\title{
A study on the relationship between the fertilization rate in IVF and the mechanical energy of sperm
}

Fumiaki Itoi ${ }^{1}$, Yukitake Tamaki ${ }^{1}$, Sachi Ohnishi ${ }^{1}$, Eri Araki ${ }^{1}$, Yumi Ogasawara ${ }^{1}$, Kana Aihara ${ }^{1}$, Hiroko Sato ${ }^{1}$, Kazuyo Nishikawa ${ }^{1}$, Masato Ohmoto $^{1}$, Yuko Otani ${ }^{1}$, Hideki Sakaguchi ${ }^{1}$ and Tetsuya Isobe ${ }^{1,2 *}$

${ }^{1}$ Green Bell ART Clinic, Kishokai Bell-net: 2-160 Kitamachi, Toyota, Aichi 471-0027, Japan

${ }^{2}$ International Oriental Medical Center, Kishokai Bell-net: 600 Hanemae, Kamishidami, Moriyama, Nagoya, Aichi 463-0001, Japan

\begin{abstract}
Purpose: The mechanical energy of sperm is an optimal index with which to quantitatively assess the motility of sperm. The sperm energy index (SEI) is proposed as an index representing the total mechanical energy of sperm in a single field when measured using computer-assisted sperm analysis (CASA). CASA systems can automatically view multiple fields in a specimen chamber to capture the images of sperm from a microscope, are capable of measuring sperm motility and kinematics using a computer. The mechanical energy of a single sperm is given by (SEI/motile sperm count) $\times 100$ which has been designated S-SEI. The aim of the current study was to examine the relationship between SEI or S-SEI and fertilization rate in conventional IVF (cIVF).
\end{abstract}

Methods: The materials used were 904 ova from 101 couples who underwent cIVF. SEI were measured in a suspension of sperm prior to insemination.

Results: Couples were classified into 4 groups based on a median SEI of 4.1 and a median S-SEI of 4.7. Couples in group D (SEI < 4.1 and S-SEI >= 4.7) had the highest rate of normal fertilization $73.9 \%$ and the lowest rate of polyspermic fertilization. Couples in group B (SEI >= 4.1 and S-SEI >= 4.7) had the highest rate of polyspermic fertilization.

Conclusions: A combination of the SEI and the S-SEI can serve as a criterion with which to determine whether couples should continue to undergo cIVF.

\section{Introduction}

The motility of sperm is activated immediately prior to fertilization in a phenomenon known as hyperactivation [1-2]. Since studies have reported this phenomenon, the motility of sperm during capacitation has been considered essential to fertilization. Computer-aided sperm analysis (CASA) has been used to obtain parameters of sperm motility [3], some studies have reported a correlation between those parameters of sperm motility and the rate of fertilization [4-7].

Currently, parameters in CASA are used as indicators of the motility of a single sperm. These include the curvilinear velocity (VCL), the amplitude of lateral head displacement (ALH), and the linearity (LIN) of the path followed by a single sperm. The percentage of motile sperm, the concentration of motile sperm, and the proportion of sperm swimming in a straight path are used as indicators of the motility of a group of sperm. However, the motility of sperm cannot be commensurately quantified on the same scale with these existing indices.

Over the past few years, laws of physics governing the forward velocity of sperm and ALH and laws of physics governing the curvature of the path followed by sperm have been defined [8-10]. The equation for sperm motility, derived from the sperm spring theory [8], and inequality of the curvature of the path followed by sperm, derived from the sperm curvature theory [9], are shown below. $A$ is the ALH, $V$ is the VCL, and $L$ is the LIN. a, b, and c are constants.

Equation for sperm motility (Sperm equation) $\left(\frac{1}{V}\right)=a\left(\frac{1}{A}\right)+b$

Inequality of the curvature of the path followed by sperm $L^{2}<1-c V^{2}$
In line with the laws of physics, the motion of an object can be commensurately quantified as its mechanical energy (the sum of its kinetic energy and potential energy). Thus, the mechanical energy of a single sperm can be used to quantitatively assess the motility of a single sperm, and the total mechanical energy of all sperm in a group is the optimal way to quantitatively assess the motility of that group.

The sperm energy index (SEI) has been derived from new sperm energy theory [11] as an index of the mechanical energy of sperm. SEI represents the total mechanical energy of the sperm existing in a single visual field of CASA. The SEI is defined as follows and can be obtained by using CASA.

$$
S E I \equiv \frac{n K \lambda}{100}
$$

Here, $\lambda$ is the mean value of the square of the ALH for all sperm in a single visual field of CASA, and $n$ is the number of motile sperm in a given field. $K$ (Isobe Coefficient) is a specific constant which fulfills the functional equation of sperm existence probability density with respect to the ALH below. Isobe coefficient can be obtained by minimizing the standard error between the actual and theoretical distribution of the probability density of sperm [12].

${ }^{*}$ Correspondence to: Tetsuya Isobe, International Oriental Medical Center, Kishokai Bell-net: 600 Hanemae, Kamishidami, Moriyama, Nagoya, Aichi 463-0001, Japan, E-mail: iso12-7@blue.ocn.ne.jp

Key words: CASA, SMAS, SEI, Sperm Energy, IVF

Received: July 06, 2018; Accepted: July 16, 2018; Published: July 20, 2018 


$$
P=\frac{C}{A\left(n \lambda-A^{2}\right)^{\frac{1}{2}+n K \lambda}}\left\{\frac{n \lambda-A^{2}}{(n-1) A^{2}}\right\}^{K A^{2}}
$$

Here, $A$ is ALH, $C$ is a constant by which $P$ value integrated by $A$ in all range becomes 1 .

The mean mechanical energy of a single sperm can be obtained by dividing SEI by the motile sperm count $n$. The single sperm energy index (S-SEI) is defined as follows:

$$
S-S E I \equiv \frac{S E I}{n} \times 100=K \lambda
$$

The aim of the current study was to use SEI and S-SEI to determine the relationship between the mechanical energy of sperm and the rate of fertilization in cIVF.

\section{Materials and method}

The materials used in this study were 904 mature ova from 101 cycles of cIVF undergone by 101 couples who were seen at this facility from September 2016 to February 2018. Metaphase II (MII) oocytes were regarded to mature ova. This study examined the relationship between the rate of normal fertilization (the proportion of ova with 2 pronuclei), the rate of polyspermic fertilization (the proportion of ova with 3 or more pronuclei), and the rate of no-fertilization (the proportion of ova with no pronuclei, NF) as a result of insemination and the SEI, which was measured in a suspension of sperm immediately prior to insemination. Couples undergoing rescue Intracytoplasmic sperm injection (ICSI) were excluded as subjects.

cIVF in this study was carried out as follows based on the report described previously by current first author [13]. The ovarian stimulation protocols included the use of gonadotrophin-releasing hormone (GnRH) antagonist (Cetrotide; Merck Serono, Tokyo, Japan) or GnRH agonist (buserelin acetate; Sprecure, Mochida Pharmaceutical) in a short protocol with recombinant follicle-stimulating hormone ( $\mathrm{rFSH}$; Gonal-F*, Merck Serono) and/or human menopausal gonadotrophin (hMG; Menopur; Ferring Pharmaceuticals, Tokyo, Japan). Another protocol was clomiphene citrate (Serophene; Merck Serono). Human chorionic gonadotrophin was administered as a single dose of 5000 IU (HCG Mochida, Mochida Pharmaceutical, Tokyo, Japan) or 300 $\mu \mathrm{g}$ of GnRH agonist was administered twice at an interval of 1 hour, at the time of $18 \mathrm{~mm}$ in the diameter of the leading follicles measured using Ultrasound. Oocyte retrieval was performed 35 hours after HCG administration or 34 hours after the second GnRH agonist dose. Oocytes were cultured using Universal IVF medium (ORIGIO Japan, Yokohama, Japan) within 3-6 hours after oocyte retrieval.

The semen sample was centrifuged with $90 \%$ Sil-select Plus (FertiPro N.V., Beemem, Belgium) at $400 \times \mathrm{g}$ for $20 \mathrm{~min}$. The sperm pellets were re-suspended and centrifuged with modified human tubal fluid (m-HTF; Irvine Scientific, CA, USA). Subsequently motile sperm were collected via the swim-up method at $37^{\circ} \mathrm{C}$. The final dilution was prepared with a sperm concentration of $0.8 \times 10^{5} / \mathrm{ml}$ using Universal IVF medium (ORIGIO Japan, Yokohama, Japan).

Parameters of sperm motility were measured immediately prior to insemination at room temperature using a newly generated sperm motility analysis system (SMAS Ver.3.00) which is a Japanese CASA system (DITECT, Tokyo, Japan). A measurement was done by using the function of continuously measuring parameters in 5 fields. Five $\mu \ell$ aliquots were placed in a Makler chamber [14] immediately prior to insemination. A Makler chamber is suitable for calculation of the SEI. The equation for sperm motility is the basis for deriving the SEI. In a previous experiment using chambers with various depths, this sperm equation was most accurately obtained with a Makler chamber [15].

The SEI was calculated by minimizing the standard error between the actual and theoretical distribution of the probability density of sperm with respect to the ALH. The SMAS allows measurements to be processed with Excel (Microsoft), so the SEI can be calculated using that Excel program (DITECT, Tokyo, Japan).

SMAS version 3 has a resolution of $52 \times 10^{5}$ pixels $(1$ pixel $=$ $0.4752761 \mu \mathrm{m}$ ) and a frame rate of $60 \mathrm{~Hz}$ (a total of 60 frames captured per second). SMAS setting were done as follows: Images were captured with a $10 \times$ objective, sperm were detected at a size of 8-80 pixels, cell intensity was 50 , and sperm were identified as immotile within a range of 4.45 pixels. The cut-off for swimming sperm was an average path velocity (VAP) of $25 \mu \mathrm{m} / \mathrm{s}$ and a straightness (STR=VSL/VAP) of $70 \%$. A hard drive containing recorded video of specimens from DITECT (Tokyo) was used for internal quality control of sperm motility.

A ratio test with a significance level of $5 \%$ was used to compare fertilization rates. An unpaired t-test with a significance level of 5\% was used to compare patient's characteristics (e.g. age).

\section{Results}

Couples were classified into 4 groups (A, B, C, and D) based on a median SEI of 4.1 and a median S-SEI of 4.7. Group A represented an SEI $>=4.7$ and an S-SEI $<4.1$, group B represented an SEI $>=4.7$ and an S-SEI $>=4.1$, group $\mathrm{C}$ represented an SEI $<4.7$ and an S-SEI $<4.1$, and group D represented an SEI $<4.7$ and an S-SEI $>=4.1$. This system of classifying patients into 4 groups was designated the bellnet classification. Definition of classification, number of mature ova of subjects, and fertilization rates for patients are shown in Table 1. The rate of fertilization for patients classified according to the bell-net classification is shown in Figure 1.

The semen volume, the concentration and percentage of motile sperm in ejaculate, the age of husbands and wives, the value of antiMüllerian hormone (AMH) and Follicle stimulating hormone (FSH) for female patients, and the number of mature oocyte per female patient in each group of the Bell-net classification are shown in Table 2. It has been reported that IVF results are significantly affected by serum AMH [16] and serum basal FSH [17]. The percent motility of sperm in ejaculate differed significantly $(\mathrm{P}=0.0029)$ among couples in groups A-D. Couples in group D had both the highest rate of normal fertilization and the lowest rate of polyspermic fertilization in spite of the lowest sperm motility in ejaculate. Significant differences in the other indices were not noted (data not shown).

Overall, the rate of normal fertilization was $72.1 \%$ (652/904), the rate of polyspermic fertilization was $11.1 \%(100 / 904)$, and the rate of non- fertilization was 9.1\% (82/904). As shown in Figure 1, the highest rate of normal fertilization was $73.9 \%$ (99/134) for couples in group $\mathrm{D}(\mathrm{SEI}<4.1$ and $\mathrm{S}-\mathrm{SEI}>=4.7)$, who had the lowest rate of polyspermic fertilization $6.0 \%(8 / 134)$. Couples in group B (SEI $>=4.1$ and S-SEI $>=4.7$ ) had the highest rate of polyspermic fertilization $14.7 \%$ $(48 / 327)$. Couples in group C $(\mathrm{SEI}<4.1, \mathrm{~S}-\mathrm{SEI}<4.7)$ had the lowest rate of normal fertilization $70.3 \%(187 / 266)$. There was a statistically significant difference $(\mathrm{P}=0.0048)$ in regard to the rate of polyspermic fertilization between couples in group $\mathrm{D}$ and couples in group $\mathrm{B}$. There was no significant difference among four groups in regard to the rate of normal fertilization. 
Table 1. Definition of classification, number of mature ova of subjects, fertilization rates for patients

\begin{tabular}{|c|c|c|c|c|c|}
\hline & A & $\mathrm{B}$ & $\mathrm{C}$ & $\mathrm{D}$ & total \\
\hline \multirow[b]{2}{*}{ Definition of classification } & $\mathrm{SEI}>=4.1$ & $\mathrm{SEI}>=4.1$ & $\mathrm{SEI}<4.1$ & $\mathrm{SEI}<4.1$ & \\
\hline & S-SEII $<4.7$ & $\mathrm{~S}-\mathrm{SEI}>=4.7$ & $\mathrm{~S}-\mathrm{SEI}<4.7$ & $\mathrm{~S}-\mathrm{SEI}>=4.7$ & \\
\hline Number of mature ova & 177 & 327 & 266 & 134 & 904 \\
\hline Number of couples & 17 & 34 & 33 & 17 & 101 \\
\hline Normal fertilization rate $(\%)$ & $72.3 \%(128 / 177)$ & $72.8 \%(238 / 327)$ & $70.3 \%(187 / 266)$ & $73.9 \%(99 / 134)$ & $72.1 \%(652 / 904)$ \\
\hline Polyspermic fertilization rate $(\%)$ & $13.6 \%(24 / 177)$ & $14.7 \%(48 / 327)$ & $7.5 \%(20 / 266)$ & $6.0 \%(8 / 134)$ & $11.1 \%(100 / 904)$ \\
\hline Non fertilization rate $(\%)$ & $9.0 \%(16 / 177)$ & $8.3 \%(27 / 327)$ & $8.3 \%(22 / 266)$ & $12.7 \%(17 / 134)$ & $9.1 \%(82 / 904)$ \\
\hline
\end{tabular}

Table 2. Characteristics of each group in Bell-net classification. The semen volume, the concentration and percentage of motile sperm in ejaculate, the age of husbands and wives, the value of anti-Müllerian hormone (AMH) and Follicle stimulating hormone (FSH) for female patients, and the number of mature oocyte per female patient in each group of the Bell-net classification are shown. The percent motility of sperm in ejaculate differed significantly $(\mathrm{P}=0.0029)$ among couples in groups A-D. Couples in group D had both the highest rate of normal fertilization and the lowest rate of polyspermic fertilization in spite of the lowest sperm motility in ejaculate. Significant differences in the other indices were not noted

\begin{tabular}{|c|c|c|c|c|}
\hline & A & $\mathrm{B}$ & $\mathrm{C}$ & $\mathrm{D}$ \\
\hline Wife's age & $35.8 \pm 4.5$ & $34.4 \pm 5.4$ & $34.9 \pm 4.4$ & $35.7 \pm 4.4$ \\
\hline AMH (ng/ml) & $5.0 \pm 3.4$ & $4.4 \pm 3.6$ & $4.5 \pm 4.8$ & $3.4 \pm 2.1$ \\
\hline FSH (mIU/ml) & $9.0 \pm 3.2$ & $9.5 \pm 3.5$ & $10.8 \pm 5.3$ & $9.9 \pm 3.9$ \\
\hline No. of mature ova/patient & $10.9 \pm 7.2$ & $10.7 \pm 5.9$ & $9.2 \pm 6.6$ & $9.4 \pm 5.9$ \\
\hline Husband's age & $35.4 \pm 5.9$ & $36.4 \pm 4.7$ & $35.8 \pm 5.2$ & $36.6 \pm 6.6$ \\
\hline Semen volume $(\mathrm{ml})$ & $3.0 \pm 1.2$ & $2.9 \pm 1.3$ & $2.9 \pm 1.5$ & $2.9 \pm 1.2$ \\
\hline Sperm concentration in ejaculate $\left(* 10^{6} / \mathrm{ml}\right)$ & $75.6 \pm 44.4$ & $63.4 \pm 33.1$ & $63.2 \pm 38.7$ & $56.9 \pm 35.2$ \\
\hline Sperm motility in ejaculate (\%) & $58.2 \pm 16.6$ & $57.4 \pm 16.6$ & $46.7 \pm 17.5$ & $39.2 \pm 20.7$ \\
\hline
\end{tabular}
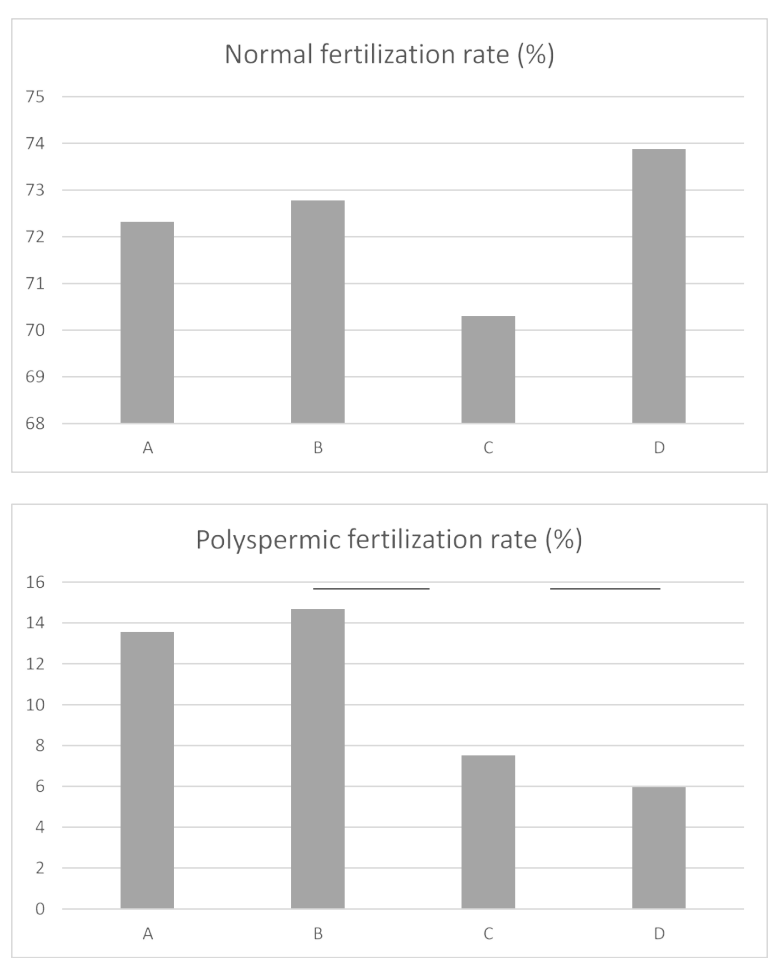

Figure 1. The rate of fertilization for patients classified according to the bell-net classification. Couples were classified into 4 groups (A, B, C, and D) based on a median SEI of 4.1 and a median S-SEI of 4.7. Group A represented an SEI $>=4.7$ and an S-SEI $<4.1$, group B represented an SEI $>=4.7$ and an S-SEI $>=4.1$, group C represented an SEI $<4.7$ and an S-SEI $<4.1$, and group D represented an SEI $<4.7$ and an S-SEI $>=4.1$. This system of classifying patients into 4 groups was designated the bell-net classification. The highest rate of normal fertilization was $73.9 \%(99 / 134)$ for couples in group D (SEI $<4.1$ and S-SEI $>=4.7)$, who had the lowest rate of polyspermic fertilization $6.0 \%(8 / 134)$. Couples in group B $(\mathrm{SEI}>=4.1$ and S-SEI $>=4.7)$ had the highest rate of polyspermic fertilization $14.7 \%$ (48/327). Couples in group C (SEI $<4.1$, S-SEI $<4.7)$ had the lowest rate of normal fertilization 70.3\% (187/266). There was a statistically significant difference $(\mathrm{P}=0.0048)$ in regard to the rate of polyspermic fertilization between couples in group $\mathrm{D}$ and couples in group $\mathrm{B}$. There was no significant difference among four groups in regard to the rate of normal fertilization

\section{Discussion}

According to the bell-net classification, group A and B have a large SEI and a high rate of polyspermic fertilization. The SEI can be expressed as the concentration of motile sperm $\times$ the mechanical energy of a single sperm, so a higher concentration of motile sperm results in more frequent contact with an ovum, thus increasing the likelihood of polyspermic fertilization. Group B and D have a large S-SEI and a high rate of normal fertilization. As the mechanical energy of a single sperm increases, sperm are more likely to pass through the zona pellucida. Group C has a small SEI, a small S-SEI, and the lowest rate of normal fertilization and a low rate of polyspermic fertilization. This may have 
been caused by sperm coming into contact with an ovum less often and sperm being unable to pass through the zona pellucida. Group D has a small SEI, a large S-SEI, the lowest rate of polyspermic fertilization, and the highest rate of normal fertilization. This may be accounted by that sperm came into contact with an ovum less often, making polyspermic fertilization less likely, but sperm that came into contact with an ovum efficiently passed through the zona pellucida. Group B had a large SEI, a large S-SEI, and the highest rate of polyspermic fertilization. This may be accounted by that the sperm with a high mechanical energy which was more likely to pass through the zona pellucida often came into contact with an ovum. According to the bell-net classification, patients falling into group $B$ have a higher risk of polyspermic fertilization. Once polyspermic fertilization has occurred, rescue ICSI is not feasible. With this in mind, a couple in case of group B should probably switch from cIVF to ICSI. The current results indicate that a combination of the SEI and the S-SEI could serve as an index with which to determine whether couples should continue to undergo cIVF or undergo ICSI instead.

A study reported that the motility of sperm was activated by adding of a methylxanthine derivative such as caffeine and pentoxifylline (PTX) [18-19]. Some studies have reported that addition of PTX improves the rate of fertilization in CIVF [20-22]. A study has reported that the motility of sperm is greatest when the concentration of PTX is $2.8 \mathrm{mM} / \mathrm{\ell}(=0.78 \mathrm{mg} / \mathrm{ml}$ : PTX has a molecular weight of $278.3 \mathrm{~g} /$ mol) after addition [23]. Studies have used the S-SEI to quantitatively assess the activation of sperm over time as a result of adding PTX to a suspension of sperm obtained via the swim-up method [24]. At room air temperature, the S-SEI decreases over time. In contrast, heating a suspension of sperm to $37^{\circ} \mathrm{C}$ results in a gradual increase in the S-SEI. After $60 \mathrm{~min}$, the S-SEI peaks and it then subsequently decreases. When PTX is added at a temperature of $37^{\circ} \mathrm{C}$ until it reaches a final concentration of $1 \mathrm{mg} / \mathrm{ml}$, the S-SEI immediately after addition increases by 1.2 in comparison to the S-SEI at room air temperature, and the mechanical energy of a single sperm increases 1.3 -fold. This effect lasts at least $90 \mathrm{~min}$.

In the future, a prospective study needs to determine whether or not polyspermic fertilization can be avoided and the rate of normal fertilization can be increased by adjusting the SEI via dilution and reconcentration or by adjusting the S-SEI via activation of sperm with PTX.

In all of the groups according to the bell-net classification, the rate of non-fertilization was higher than $8.3 \%$. Despite having increased motility, a certain number of sperm failed to fertilize an ovum. This indicates that fertilization is not determined solely by the motility of sperm. Other factors for fertilization besides the motility of sperm are enzymatic activity in the acrosome on the head of sperm and the quality of ova. Indices should appear in the future which will combine the SEI, the S-SEI, and the parameters to determine the biological ability of sperm to fertilize an ovum, and the quality of ova.

\section{Disclosures}

This study was conducted in compliance with the Helsinki Declaration. This research project has been approved by a suitably constituted Ethics Committee. This study was approved by the Institutional review board of this facility. All patients provided consent to all treatment procedures and agreed to anonymous use of their data for studies, and obtained data were kept secure at this facility in a form in which patients could not be identified individually.

The current authors had no conflicts of interest with regard to this study. This research did not receive any specific grant from any funding agency in the public, commercial or not-for-profit sector.

\section{References}

1. Morales P, Overstreet JW, Katz DF (1988) Changes in human sperm motion during capacitation in vitro. J Reprod Fertil 83: 119-128. [Crossref]

2. Yanagimachi R (1970) The movement of golden hamster spermatozoa before and after capacitation. J Reprod Fertil 23: 193-196. [Crossref]

3. 5th ed. Geneva: World Health Organization; 2010. World Health Organization. WHO laboratory Manual for the Examination and Processing of Human Semen; p139.

4. Larsen L, Scheike T, Jensen TK, Bonde JP, Emst E, et al. (2000) Computer-assisted semen analysis parameters as predictors for fertility of men from the general population. Hum Reprod, 15:1562-1567. [Crossref]

5. Fetterolf PM, Rogers BJ (1990) Prediction of human sperm penetrating ability using computerized motion parameters. Mol Reprod Dev. 27: 326-331. [Crossref]

6. King LM, Holsberger DR, Donoghue AM (2000) Correlation of CASA velocity and linearity parameters with sperm mobility phenotype in turkeys. $J$ Androl 21: 65-71. [Crossref]

7. Kathiravan P, Kalatharan J, John EM, Veerapandian C (2008) Computer automated motion analysis of crossbred bull spermatozoa and its relationship with in vitro fertility in zona-free hamster oocytes. Anim Reprod Sci. 104: 9-17. [Crossref]

8. Isobe T (2007) Mathematical Analysis of Sperm Motility. Japan Society of Fertil Implant. 24: 6-15.

9. Isobe T, Matsuura D (2008) Examination of Sperm Curvature using CASA. Japan Society of Fertil Implant. 25: 6-11.

10. Isobe T (2014) New Perspective Regarding the Sperm. J Urol Res. 1: 1004-1005.

11. Isobe $\mathrm{T}$ (2012) New method to estimate the possibility of natural pregnancy using computer-assisted sperm analysis. Syst Biol Reprod Med. 58: 339-347. [Crossref]

12. Isobe T (2016) How to Determine an Index of the Mechanical Energy of Sperm. Austin J Urol. 3: 1-4.

13. Itoi F, Asano Y, Shimizu M, Honnma H, Murata Y (2016) Embryological outcomes in cycles with human oocytes containing large tubular smooth endoplasmic reticulum clusters after conventional in vitro fertilization. Gynecol. Endocrinol. 32: 315-318. [Crossref]

14. Makler A (1980) The improved ten-micrometer chamber for rapid sperm count and motility evaluation. Fertil Steril 33: 337-338. [Crossref]

15. Isobe $\mathrm{T}$ (2008) Assessment of fertility by sperm mechanical energy using computerassisted sperm analysis system. Reprod Med Biol 8: 25-31. [Crossref]

16. Revelli A, Biasoni V, Gennarelli G, Canosa S, Dalmasso P, et al. (2016) IVF results in patients with very low serum AMH are significantly affected by chronological age. $J$ Assist Reprod Genet 33: 603-609. [Crossref]

17. Abdalla H, Thum MY (2004) An elevated basal FSH reflects a quantitative rather than qualitative decline of the ovarian reserve. Hum Reprod 19: 893-898. [Crossref]

18. Dimitriadou F, Voutsina K, Rizos D, Prapa A, Mantzavinos T, et.al. (1995) The effect of pentoxifylline on sperm motility, oocyte fertilization, embryo quality, and pregnancy outcome in an in vitro fertilization program. Fertil Steril. 63: 880-886. [Crossref]

19. Guasti NP, Monteiro AG, Maziero RR, Martin I, Avanzi RB, et al. (2013) Effects of Pentoxifylline on Equine Epididymal Sperm. J Equine Veterinary Science. 33: 11531156.

20. Fountain S, Rizk B, Avery S, Palmer C, Blayney M, et al. (1995) An evaluation of the effect of pentoxifylline on sperm function and treatment outcome of male-factor infertility: a preliminary study. J Assist Reprod Genet 12: 704-709. [Crossref]

21. Tarlatzis BC, Kolibianakis EM, Bontis J, Tousiou M, Lagos S, et al. (1995) Effect of pentoxifylline on human sperm motility and fertilizing capacity. Arch Androl 34: 3342. [Crossref]

22. Rizk B, Fountain S, Avery S, Palmer C, Blayney M, et al. (1995) Successful use of pentoxifylline in male-factor infertility and previous failure of in vitro fertilization: a prospective randomized study. J Assisted Genet. 12: 710-4. [Crossref]

23. Paul M, Sumpter JP, Lindsay KS (1996) Factors affecting pentoxifylline stimulation of sperm kinematics in suspensions. Hum reprod, 11: 1929-1935. [Crossref]

24. Ohnishi S, Itoi F, Tamaki Y, Nishikawa K, Isobe T (2018) Quantitative Evaluation of Effect of Incubation or addition of Pentoxifylline on Sperm Activation . Japan Society of Fertil Implant. 35: 163-168.

Copyright: (C2018 Itoi F. This is an open-access article distributed under the terms of the Creative Commons Attribution License, which permits unrestricted use, distribution, and reproduction in any medium, provided the original author and source are credited. 